\title{
MacMahon's partition analysis XI: Broken diamonds and modular forms
}

\author{
by
}

George E. Andrews (University Park, PA) and Peter Paule (Linz)

1. Introduction. In his pioneering book "Combinatory Analysis" [13, Vol. II, Sect. VIII, pp. 91-170] MacMahon introduced Partition Analysis as a computational method for solving combinatorial problems in connection with systems of linear diophantine inequalities and equations. In particular, he devotes Chapter II of Section IX to the study of plane partitions as a natural application domain for his method.

In the course of a joint project devoted to Partition Analysis, the authors have turned MacMahon's method into an algorithm described in full detail in $[5,6]$. As demonstrated in references [2]-[11], the resulting computer algebra package Omega $\left(^{1}\right)$ has been used as a powerful tool for combinatorial investigation. In particular, in [8] we introduced "partition diamonds" as new variations of plane partitions. This work inspired various other extensions; see, for instance, [12] and [10]. Also the present paper can be viewed as a generalization of [8] but being completely different from [12] and [10]. The graphs considered below are made up of chains of elongated partition diamonds, and the related generating functions are infinite products. The culmination of our study leads to an infinite family of modular forms. These, in turn, lead to interesting arithmetic theorems and conjectures for the related partition functions.

The "most simple case" of classical plane partitions, treated by MacMahon in [13, Vol. II, p. 183], is the situation where the non-negative integer parts $a_{i}$ of the partitions are placed at the corners of a square such that the following order relations are satisfied:

$$
a_{1} \geq a_{2}, \quad a_{1} \geq a_{3}, \quad a_{2} \geq a_{4}, \quad a_{3} \geq a_{4} .
$$

2000 Mathematics Subject Classification: 05A15, 05A17, 11P83.

The first author was partially supported by NSF grant DMS-0200047.

The second author was partially supported by SFB grant F1305 of the Austrian FWF.

${ }^{1}$ ) http://www.risc.uni-linz.ac.at/research/combinat/risc/software/Omega/. 
It will be convenient to use arrows as an alternative description for $\geq$ relations; for instance, Fig. 1 represents the relations (1.1). Here and throughout the following it will be understood that an arrow pointing from $a_{i}$ to $a_{j}$ is interpreted as $a_{i} \geq a_{j}$.

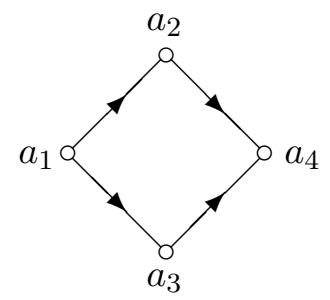

Fig. 1. The inequalities (1.1)

By using Partition Analysis MacMahon derives that

$$
\begin{aligned}
\varphi & :=\sum x_{1}^{a_{1}} x_{2}^{a_{2}} x_{3}^{a_{3}} x_{4}^{a_{4}} \\
& =\frac{1-x_{1}^{2} x_{2} x_{3}}{\left(1-x_{1}\right)\left(1-x_{1} x_{2}\right)\left(1-x_{1} x_{3}\right)\left(1-x_{1} x_{2} x_{3}\right)\left(1-x_{1} x_{2} x_{3} x_{4}\right)},
\end{aligned}
$$

where the sum is taken over all non-negative integers $a_{i}$ satisfying (1.1). Furthermore, he observes that if $x_{1}=x_{2}=x_{3}=x_{4}=q$, the resulting generating function is

$$
\frac{1}{(1-q)\left(1-q^{2}\right)^{2}\left(1-q^{3}\right)} .
$$

In order to see how Partition Analysis works on (1.2) we need to recall the key ingredient of MacMahon's method, the Omega operator $\Omega_{\geq}$.

DeFinition 1. The operator $\Omega_{\geq}$is given by

$$
\Omega_{\geq} \sum_{s_{1}=-\infty}^{\infty} \cdots \sum_{s_{r}=-\infty}^{\infty} A_{s_{1}, \ldots, s_{r}} \lambda_{1}^{s_{1}} \cdots \lambda_{r}^{s_{r}}:=\sum_{s_{1}=0}^{\infty} \cdots \sum_{s_{r}=0}^{\infty} A_{s_{1}, \ldots, s_{r}},
$$

where the domain of the $A_{s_{1}, \ldots, s_{r}}$ is the field of rational functions over $\mathbb{C}$ in several complex variables and the $\lambda_{i}$ are restricted to a neighborhood of the circle $\left|\lambda_{i}\right|=1$. In addition, the $A_{s_{1}, \ldots, s_{r}}$ are required to be such that any of the series involved is absolutely convergent within the domain of the definition of $A_{s_{1}, \ldots, s_{r}}$.

To avoid confusion we will always have $\Omega_{\geq}$operate on variables denoted by letters in the middle of the Greek alphabet (e.g. $\lambda, \mu, \nu$ ). The parameters unaffected by $\Omega_{\geq}$will be denoted by letters from the Latin alphabet.

We emphasize that it is essential to treat everything analytically rather than formally because the method relies on unique Laurent series representations of rational functions. 
Another fundamental aspect of Partition Analysis is the use of elimination rules which describe the action of the Omega operator on certain base cases. MacMahon begins the discussion of his method by presenting a catalog [13, Vol. II, pp. 102-103] of twelve fundamental evaluations. Subsequently he extends this table by new rules whenever he is forced to do so. Once found, most of these fundamental rules are easy to prove. This is illustrated by the following examples which are taken from MacMahon's list.

Proposition 1. For each integer $s \geq 1$,

$$
\begin{aligned}
& \Omega_{\geq} \frac{1}{(1-\lambda A)\left(1-B / \lambda^{s}\right)}=\frac{1}{(1-A)\left(1-A^{s} B\right)} \\
& \Omega_{\geq} \frac{1}{(1-\lambda A)(1-\lambda B)(1-C / \lambda)}=\frac{1-A B C}{(1-A)(1-B)(1-A C)(1-B C)} .
\end{aligned}
$$

Proof. We prove (1.3); the proof of (1.4) is analogous and is left to the reader. By geometric series expansion the left-hand side equals

$$
\Omega_{\geq} \sum_{i, j \geq 0} \lambda^{i-s j} A^{i} B^{j}=\Omega_{\geq} \sum_{j, k \geq 0} \lambda^{k} A^{s j+k} B^{j},
$$

where the summation parameter $i$ has been replaced by $s j+k$. But now $\Omega_{\geq}$ sets $\lambda$ to 1 , which completes the proof.

Now we are ready for deriving the closed form expression for $\varphi$ with Partition Analysis.

Proof of (1.2). First, in order to get rid of the diophantine constraints, one rewrites the sum expression in (1.2) into what MacMahon called the "crude form" of the generating function,

$$
\begin{aligned}
\varphi & =\Omega_{\geq} \sum_{a_{1}, a_{2}, a_{3}, a_{4} \geq 0} \lambda_{1}^{a_{1}-a_{2}} \lambda_{2}^{a_{1}-a_{3}} \lambda_{3}^{a_{2}-a_{4}} \lambda_{4}^{a_{3}-a_{4}} x_{1}^{a_{1}} x_{2}^{a_{2}} x_{3}^{a_{3}} x_{4}^{a_{4}} \\
& =\Omega_{\geq} \frac{1}{\left(1-\lambda_{1} \lambda_{2} x_{1}\right)\left(1-\lambda_{3} x_{2} / \lambda_{1}\right)\left(1-\lambda_{4} x_{3} / \lambda_{2}\right)\left(1-x_{4} /\left(\lambda_{3} \lambda_{4}\right)\right)} .
\end{aligned}
$$

Next by rule (1.3) we eliminate successively $\lambda_{1}, \lambda_{3}$, and $\lambda_{4}$,

$$
\begin{aligned}
\varphi & =\Omega_{\geq} \frac{1}{\left(1-\lambda_{2} x_{1}\right)\left(1-\lambda_{2} \lambda_{3} x_{1} x_{2}\right)\left(1-\lambda_{4} x_{3} / \lambda_{2}\right)\left(1-x_{4} /\left(\lambda_{3} \lambda_{4}\right)\right)} \\
& =\Omega_{\geq} \frac{1}{\left(1-\lambda_{2} x_{1}\right)\left(1-\lambda_{2} x_{1} x_{2}\right)\left(1-\lambda_{4} x_{3} / \lambda_{2}\right)\left(1-\lambda_{2} x_{1} x_{2} x_{4} / \lambda_{4}\right)} \\
& =\Omega_{\geq} \frac{1}{\left(1-\lambda_{2} x_{1}\right)\left(1-\lambda_{2} x_{1} x_{2}\right)\left(1-x_{3} / \lambda_{2}\right)\left(1-x_{1} x_{2} x_{3} x_{4}\right)} .
\end{aligned}
$$

Finally, applying rule (1.4) eliminates $\lambda_{2}$ and completes the proof of (1.2). 
Instead of glueing squares together as in the case of standard plane partitions, in [8] we considered configurations shown in Fig. 2. In the present

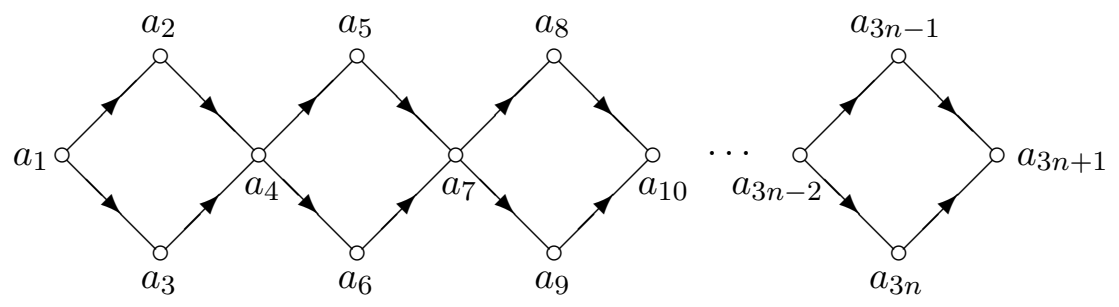

Fig. 2. A plane partition diamond of length $n$

paper we shall study the natural generalization depicted in Fig. 4 where we use $k$-elongated diamonds, depicted in Fig. 3, instead of squares as building blocks of the chain.

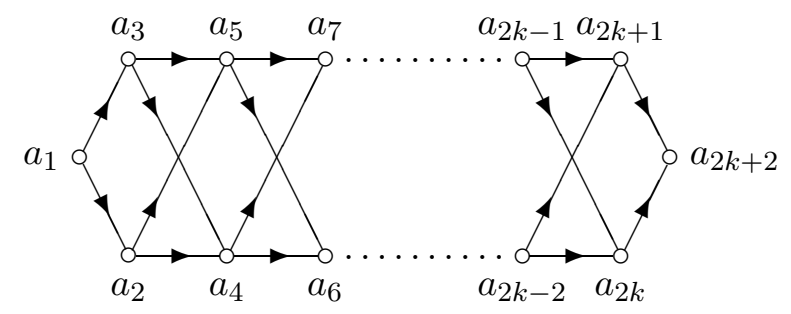

Fig. 3. A $k$-elongated partition diamond of length 1

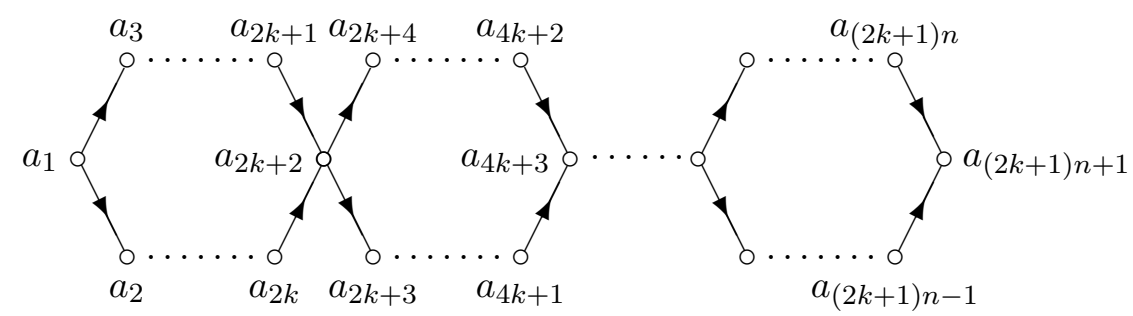

Fig. 4. A $k$-elongated partition diamond of length $n$

Definition 2. For $n, k \geq 1$ define

$$
H_{n, k}:=\left\{\left(a_{1}, \ldots, a_{(2 k+1) n+1}\right) \in \mathbb{N}^{(2 k+1) n+1}:\right.
$$

the $a_{i}$ satisfy the order relations in Fig. 4\},

$$
h_{n, k}:=h_{n, k}\left(x_{1}, \ldots, x_{(2 k+1) n+1}\right):=\sum_{\left(a_{1}, \ldots, a_{(2 k+1) n+1}\right) \in H_{n, k}} x_{1}^{a_{1}} \cdots x_{(2 k+1) n+1}^{a_{(2 k+1) n+1}},
$$

$h_{n, k}(q):=h_{n, k}(q, \ldots, q)$. 
In Section 2 we shall derive a closed form, Theorem 6, for the full generating function $h_{n, k}$. As a corollary, we will directly deduce

TheOREM 1. For $n, k \geq 1$,

$$
h_{n, k}(q)=\frac{\prod_{j=0}^{n-1}\left(1+q^{(2 k+1) j+2}\right)\left(1+q^{(2 k+1) j+4}\right) \cdots\left(1+q^{(2 k+1) j+2 k}\right)}{\prod_{j=1}^{(2 k+1) n+1}\left(1-q^{j}\right)} .
$$

In Section 3, we shall prove a general theorem about partitions related to directed graphs from which a source is deleted. In Figures 2, 3 and $4, a_{1}$ is a unique source.

Definition 3. For $n, k \geq 1$ define

$$
\begin{aligned}
H_{n, k}^{*}:=\left\{\left(a_{2}, \ldots, a_{(2 k+1) n+1}\right)\right. & \in \mathbb{N}^{(2 k+1) n}: \\
& \text { the } a_{i} \text { satisfy the order relations in Fig. } 4 \\
& \text { where the vertex labeled } \left.a_{1} \text { has been deleted }\right\},
\end{aligned}
$$

$$
\begin{aligned}
h_{n, k}^{*} & :=h_{n, k}^{*}\left(x_{2}, \ldots, x_{(2 k+1) n+1}\right):=\sum_{\left(a_{2}, \ldots, a_{(2 k+1) n+1} \in H_{n, k}^{*}\right.} x_{2}^{a_{2}} \cdots x_{(2 k+1) n+1}^{a_{(2 k+1) n+1}}, \\
h_{n, k}^{*}(q) & :=h_{n, k}^{*}(q, \ldots, q) .
\end{aligned}
$$

In Section 4 we shall derive (Theorem 8) a closed form for the full generating function $h_{n, k}^{*}$, and from this we shall prove

Theorem 2. For $n, k \geq 1$,

$$
h_{n, k}^{*}(q)=\frac{\prod_{j=0}^{n-1}\left(1+q^{(2 k+1) j+1}\right)\left(1+q^{(2 k+1) j+3}\right) \cdots\left(1+q^{(2 k+1) j+2 k-1}\right)}{\prod_{j=1}^{(2 k+1) n}\left(1-q^{j}\right)} .
$$

This then suggests the broken $k$-diamond in Fig. 5 ; it consists of two separated $k$-elongated partition diamonds of length $n$ where in one of them the source is deleted.

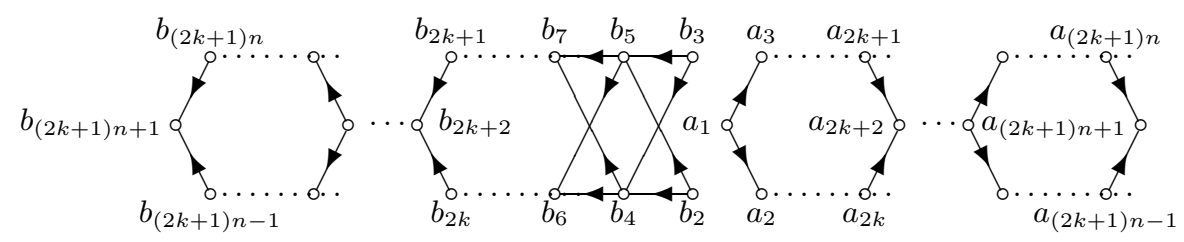

Fig. 5. A broken $k$-diamond of length $2 n$ 
Definition 4. For $n, k \geq 1$ define

$$
\begin{aligned}
H_{n, k}^{\diamond} & :=\left\{\left(b_{2}, \ldots, b_{(2 k+1) n+1}, a_{1}, \ldots, a_{(2 k+1) n+1}\right) \in \mathbb{N}^{(4 k+2) n+1}:\right. \\
& \text { the } \left.a_{i} \text { and } b_{i} \text { satisfy all the order relations in Fig. } 5\right\}, \\
h_{n, k}^{\diamond} & :=h_{n, k}^{\diamond}\left(x_{2}, \ldots, x_{(2 k+1) n+1} ; y_{1}, \ldots, y_{(2 k+1) n+1}\right) \\
& :=\sum_{\left(b_{2}, \ldots, b_{(2 k+1) n+1}, a_{1}, \ldots, a_{(2 k+1) n+1}\right) \in H_{n, k}^{\diamond}}^{b_{2}} \cdots x_{(2 k+1) n+1}^{b_{(2 k+1) n+1}} y_{1}^{a_{1}} \cdots y_{(2 k+1) n+1}^{a_{(2 k+1) n+1}}, \\
h_{n, k}^{\diamond}(q) & :=h_{n, k}^{\diamond}(q, \ldots, q) .
\end{aligned}
$$

Now it is immediate from the fact that Fig. 5 is made up of two disconnected directed graphs that

$$
h_{n, k}^{\diamond}=h_{n, k} h_{n, k}^{*}
$$

Owing to Theorems 1 and 2 this immediately implies

TheOREM 3. For $k \geq 1$,

$$
h_{\infty, k}^{\diamond}(q)=\prod_{j=1}^{\infty} \frac{1+q^{j}}{\left(1-q^{j}\right)^{2}\left(1+q^{(2 k+1) j}\right)}=\frac{q^{(k+1) / 12} \eta(2 \tau) \eta((2 k+1) \tau)}{\eta(\tau)^{3} \eta((4 k+2) \tau)},
$$

where $q=e^{2 \pi i \tau}$, and $\eta(\tau):=q^{1 / 24} \prod_{n=1}^{\infty}\left(1-q^{n}\right)$ is Dedekind's $\eta$-function.

Definition 5. For $n \geq 0$ and $k \geq 1$ let $\Delta_{k}(n)$ denote the total number of broken $k$-diamond partitions of $n$, i.e.,

$$
h_{\infty, k}^{\diamond}(q)=\sum_{n=0}^{\infty} \Delta_{k}(n) q^{n}
$$

In Section 5 we shall prove the following two theorems.

Theorem 4. For $n, k \geq 1$,

$$
\Delta_{k}(n)+2 \sum_{j=1}^{\infty}(-1)^{j} \Delta_{k}\left(n-j^{2}\right)
$$

is equal to the number of ordinary partitions of $n$ into parts not congruent to $2 k+1$ modulo $4 k+2$.

TheOREM 5. For $n \geq 0$,

$$
\Delta_{1}(2 n+1) \equiv 0(\bmod 3) .
$$

The following observations about congruences suggest strongly that there are undoubtedly a myriad of partition congruences for $\Delta_{k}(n)$. This list is only to indicate the tip of the iceberg.

Conjecture 1 . For $n \geq 0$,

$$
\Delta_{2}(10 n+2) \equiv 0(\bmod 2) \text {. }
$$


Conjecture 2. For $n \geq 0$,

$$
\Delta_{2}(25 n+14) \equiv 0(\bmod 5) .
$$

Conjecture 3. For $n \geq 0$, if 3 does not divide $n$ then

$$
\Delta_{2}(625 n+314) \equiv 0\left(\bmod 5^{2}\right) \text {. }
$$

2. A closed form for $h_{n, k}$. In this section we shall prove the following theorem which provides a representation of the generating function $h_{n, k}$ as a product. Throughout we shall use the convention

$$
X_{0}:=1, \quad X_{m}:=x_{1} \cdots x_{m} \quad(m \geq 1) .
$$

Theorem 6 . For $n, k \geq 1$,

$$
\begin{aligned}
h_{n, k}\left(x_{1}, \ldots, x_{(2 k+1) n+1}\right) \\
=\prod_{j=1}^{(2 k+1) n+1} \frac{1}{1-X_{j}} \prod_{i=0}^{n-1} \prod_{l=1}^{k} \frac{1-X_{(2 k+1) i+2 l-1} X_{(2 k+1) i+2 l+1}}{1-X_{(2 k+1) i+2 l+1} / x_{(2 k+1) i+2 l}} .
\end{aligned}
$$

The proof of Theorem 6 requires some background preparation. Consider the $k$-elongated partition diamond of length $n=1$ in Figure 3. Similarly to the proof of (1.2), the inequalities represented by the arrows can be coded into the form

$$
\begin{aligned}
\Lambda\left(\lambda_{1}, \ldots, \lambda_{4 k}\right):= & \lambda_{1}^{a_{1}-a_{2}} \lambda_{2}^{a_{1}-a_{3}} \lambda_{3}^{a_{2}-a_{4}} \lambda_{4}^{a_{2}-a_{5}} \lambda_{5}^{a_{3}-a_{4}} \lambda_{6}^{a_{3}-a_{5}} \times \cdots \\
& \times \lambda_{4 k-5}^{a_{2 k-2}-a_{2 k}} \lambda_{4 k-4}^{a_{2 k-2}-a_{2 k+1}} \lambda_{4 k-3}^{a_{2 k-1}-a_{2 k}} \lambda_{4 k-2}^{a_{2 k-1}-a_{2 k+1}} \\
& \times \lambda_{4 k-1}^{a_{2 k}-a_{2 k+2}} \lambda_{4 k}^{a_{2 k+1}-a_{2 k+2}} .
\end{aligned}
$$

We shall use this notation subsequently in our treatment of the case $n=1$ of Theorem 6; see Lemma 2.2 and its proof.

The key to our Partition Analysis proof of Theorem 6 is the following elimination rule.

LEMMA 2.1.

$$
\begin{array}{r}
\Omega_{\geq} \frac{1-A B \lambda_{1} \lambda_{2}}{\left(1-A \lambda_{1}\right)\left(1-B \lambda_{2}\right)\left(1-C \lambda_{1} \lambda_{2}\right)\left(1-D \lambda_{1} \lambda_{2}\right)\left(1-E /\left(\lambda_{1} \lambda_{2}\right)\right)} \\
=\frac{(1-A B)(1-C D E)}{(1-A)(1-B)(1-C)(1-D)(1-C E)(1-D E)} .
\end{array}
$$

We remark that (2.3) may be proved automatically using the Omega package. A direct proof is also easy to produce. First prove the case $D=0$ of (2.3) by successively applying rule (1.4) and the similar rule [6, eq. (2.2)]

$$
\Omega_{\geq} \frac{\lambda}{(1-A \lambda)(1-B \lambda)(1-C / \lambda)}=\frac{1+C-A C-B C}{(1-A)(1-B)(1-A C)(1-B C)} .
$$


Lemma 2.1 then follows from the case $D=0$ of (2.3) after rewriting the left-hand side of (2.3) by inserting the partial fraction decomposition

$$
\frac{1}{\left(1-C \lambda_{1} \lambda_{2}\right)\left(1-D \lambda_{1} \lambda_{2}\right)}=\frac{C}{(C-D)\left(1-C \lambda_{1} \lambda_{2}\right)}-\frac{D}{(C-D)\left(1-D \lambda_{1} \lambda_{2}\right)} .
$$

Our proof of Theorem 6 will be by mathematical induction on $n$. We state the initial $n=1$ case as a separate lemma.

Lemma 2.2. For $k \geq 1$,

$$
h_{1, k}\left(x_{1}, \ldots, x_{2 k+2}\right)=\prod_{j=1}^{2 k+2} \frac{1}{1-X_{j}} \prod_{l=1}^{k} \frac{1-X_{2 l-1} X_{2 l+1}}{1-X_{2 l+1} / x_{2 l}} .
$$

Proof. We now proceed by induction on $k$. The case $k=1$ is identity (1.2). To pass from step $k$ to step $k+1$, we note that

$$
\begin{aligned}
& h_{1, k+1}\left(x_{1}, \ldots, x_{2 k+4}\right) \\
&=\Omega_{\geq} \sum_{a_{i} \geq 0} x_{1}^{a_{1}} \cdots x_{2 k}^{a_{k}} x_{2 k+1}^{a_{2 k+1}} x_{2 k+2}^{a_{2 k+2}} x_{2 k+3}^{a_{2 k+3}} x_{2 k+4}^{a_{2 k+4}} \\
& \times \Lambda\left(\lambda_{1}, \ldots, \lambda_{4 k}\right) \lambda_{4 k+1}^{a_{2 k}-a_{2 k+3}} \lambda_{4 k+2}^{a_{2 k+1}-a_{2 k+3}} \lambda_{4 k+3}^{a_{2 k+2}-a_{2 k+4}} \lambda_{4 k+4}^{a_{2 k+3}-a_{2 k+4}} \\
&= \Omega_{\geq} \sum_{a_{1}, \ldots, a_{2 k+2 \geq 0}} x_{1}^{a_{1}} \cdots x_{2 k-1}^{a_{2 k-1}}\left(x_{2 k} \lambda_{4 k+1}\right)^{a_{2 k}}\left(x_{2 k+1} \lambda_{4 k+2}\right)^{a_{2 k+1}} \\
& \times\left(x_{2 k+2} \lambda_{4 k+3}\right)^{a_{2 k+2}} \Lambda\left(\lambda_{1}, \ldots, \lambda_{4 k}\right) \\
& \times \sum_{a_{2 k+3}, a_{2 k+4} \geq 0}\left(x_{2 k+3} \frac{\lambda_{4 k+4}}{\lambda_{4 k+1} \lambda_{4 k+2}}\right)^{a_{2 k+3}}\left(\frac{x_{2 k+4}}{\lambda_{4 k+3} \lambda_{4 k+4}}\right)^{a_{2 k+4}} \\
&= \Omega_{\geq} \frac{h_{1, k}\left(x_{1}, \ldots, x_{2 k-1}, x_{2 k} \mu_{1}, x_{2 k+1} \mu_{2}, x_{2 k+2} \mu_{3}\right)}{\left(1-x_{2 k+3} \mu_{4} /\left(\mu_{1} \mu_{2}\right)\right)\left(1-x_{2 k+4} /\left(\mu_{3} \mu_{4}\right)\right)}
\end{aligned}
$$

where for brevity we have written $\mu_{i}$ instead of $\lambda_{4 k+i}$ in the last line. We now apply the induction hypothesis and obtain

$$
\begin{aligned}
h_{1, k+1}\left(x_{1}, \ldots, x_{2 k+4}\right) & \\
= & \prod_{j=1}^{2 k-1} \frac{1}{1-X_{j}} \prod_{l=1}^{k-1} \frac{1-X_{2 l-1} X_{2 l+1}}{1-X_{2 l+1} / x_{2 l}} \\
& \times \Omega_{\geq} \frac{1}{\left(1-X_{2 k} \mu_{1}\right)\left(1-X_{2 k+1} \mu_{1} \mu_{2}\right)\left(1-X_{2 k+2} \mu_{1} \mu_{2} \mu_{3}\right)} \\
& \times \frac{1-X_{2 k-1} X_{2 k+1} \mu_{1} \mu_{2}}{1-X_{2 k+1} \mu_{2} / x_{2 k}} \cdot \frac{1}{\left(1-x_{2 k+3} \frac{\mu_{4}}{\mu_{1} \mu_{2}}\right)\left(1-x_{2 k+4} /\left(\mu_{3} \mu_{4}\right)\right)} .
\end{aligned}
$$

Eliminating $\mu_{3}$ and $\mu_{4}$ by rule (1.3) with $s=1$, the $\Omega_{\geq}$portion of the 
above expression reduces to

$$
\begin{aligned}
\frac{1}{1-X_{2 k+4}} \Omega_{\geq} & \frac{1-X_{2 k-1} X_{2 k+1} \mu_{1} \mu_{2}}{\left(1-X_{2 k} \mu_{1}\right)\left(1-X_{2 k+1} \mu_{2} / x_{2 k}\right)} \\
& \times \frac{1}{\left(1-X_{2 k+1} \mu_{1} \mu_{2}\right)\left(1-X_{2 k+2} \mu_{1} \mu_{2}\right)\left(1-x_{2 k+3} /\left(\mu_{1} \mu_{2}\right)\right)} \\
= & \prod_{j=2 k}^{2 k+4} \frac{1}{1-X_{j}} \cdot \frac{\left(1-X_{2 k-1} X_{2 k+1}\right)\left(1-X_{2 k+1} X_{2 k+3}\right)}{\left(1-X_{2 k+1} / x_{2 k}\right)\left(1-X_{2 k+3} / x_{2 k+2}\right)},
\end{aligned}
$$

where the last line follows by Lemma 2.1. This then completes the proof of Lemma 2.2 by induction.

Proof of Theorem 6 . We proceed by induction on $n$. The case $n=1$ is Lemma 2.2. For the induction step we proceed similarly to the proof of Lemma 2.2. Namely,

$$
\begin{aligned}
& h_{n+1, k}\left(x_{1}, \ldots, x_{(2 k+1) n+1}, x_{(2 k+1) n+2}, \ldots, x_{(2 k+1) n+2 k+2}\right) \\
& =\Omega_{\geq} \sum_{a_{i} \geq 0} x_{1}^{a_{1}} \cdots x_{(2 k+1) n+1}^{a_{(2 k+1) n+1}} x_{(2 k+1) n+2}^{a_{(2 k+1) n+2}} \cdots x_{(2 k+1) n+2 k+2}^{a_{(2 k+1) n+2 k+2}} \\
& \times \lambda_{1}^{a_{1}-a_{2}} \cdots \lambda_{4 k n}^{a_{(2 k+1) n}-a_{(2 k+1) n+1}} \lambda_{4 k n+1}^{a_{(2 k+1) n+1}-a_{(2 k+1) n+2}} \\
& \times \lambda_{4 k n+2}^{a_{(2 k+1) n+1}-a_{(2 k+1) n+3}} \cdots \lambda_{4 k(n+1)}^{a_{(2 k+1) n+2 k+1}-a_{(2 k+1) n+2 k+2}} \\
& =\Omega_{\geq} h_{n, k}\left(x_{1}, \ldots, x_{(2 k+1) n}, x_{(2 k+1) n+1} \mu_{1} \mu_{2}\right) \\
& \times \sum_{b_{2}, \ldots, b_{2 k+2 \geq 0}} x_{(2 k+1) n+2}^{b_{2}} \cdots x_{(2 k+1) n+2 k+2}^{b_{2 k+2}} \mu_{1}^{0-b_{2}} \mu_{2}^{0-b_{3}} \cdots \mu_{4 k}^{b_{2 k+1}-b_{2 k+2}},
\end{aligned}
$$

where again for brevity we have written $b_{i}$ for $a_{(2 k+1) n+i}$, and $\mu_{i}$ for $\lambda_{4 k n+i}$. We now apply the induction hypothesis and obtain

$$
\begin{aligned}
& h_{n+1, k}\left(x_{1}, \ldots, x_{(2 k+1)(n+1)+1}\right) \\
& =\prod_{j=1}^{(2 k+1) n} \frac{1}{1-X_{j}} \prod_{i=0}^{n-1} \prod_{l=1}^{k} \frac{1-X_{(2 k+1) i+2 l-1} X_{(2 k+1) i+2 l+1}}{1-X_{(2 k+1) i+2 l+1} / x_{(2 k+1) i+2 l}} \\
& \quad \times \Omega_{\geq} \frac{1}{1-X_{(2 k+1) n+1} \mu_{1} \mu_{2}} \sum_{b_{2}, \ldots, b_{2 k+2 \geq 0}} x_{(2 k+1) n+2}^{b_{2}} \cdots x_{(2 k+1) n+2 k+2}^{b_{2 k+2}} \\
& \quad \times \mu_{1}^{0-b_{2}} \mu_{2}^{0-b_{3}} \cdots \mu_{4 k}^{b_{2 k+1}-b_{2 k+2}} .
\end{aligned}
$$

Noting by the geometric series that

$$
\frac{1}{1-X_{(2 k+1) n+1} \mu_{1} \mu_{2}}=\sum_{b_{1} \geq 0} X_{(2 k+1) n+1}^{b_{1}} \mu_{1}^{b_{1}} \mu_{2}^{b_{2}},
$$


we see that the above $\Omega_{\geq}$expression becomes

$$
h_{1, k}\left(X_{(2 k+1) n+1}, x_{(2 k+1) n+2}, \ldots, x_{(2 k+1) n+2 k+2}\right),
$$

which by Lemma 2.2 equals

$$
\prod_{j=(2 k+1) n+1}^{(2 k+1)(n+1)+1} \frac{1}{1-X_{j}} \prod_{l=1}^{k} \frac{1-X_{(2 k+1) n+2 l-1} X_{(2 k+1) n+2 l+1}}{1-X_{(2 k+1) n+2 l+1} / x_{(2 k+1) n+2 l}} .
$$

This completes the induction step and thus the proof of Theorem 6 .

Proof of Theorem 1. In Theorem 6 replace each $x_{i}$ with $q$.

3. Source deletion. We propose here to prove a general theorem that we shall subsequently apply to the $k$-diamonds considered in Section 2 .

We now consider a general directed graph $\mathcal{D}$ with $N$ vertices $v_{1}, \ldots, v_{N}$. As in the special cases considered in Section 1, we associate partitions by considering as parts non-negative integers $a_{i}$ placed at each vertex $v_{i}$, with the understanding that the direction arrows between vertices are interpreted as " $\geq$ " between the related summands.

Define the associated generating function by

$$
\mathcal{P}(\mathcal{D})=\sum x_{1}^{a_{1}} \cdots x_{N}^{a_{N}}
$$

where the sum is over all partitions associated with $\mathcal{D}$.

TheOREM 7. Suppose $v_{1}$ is a source in $\mathcal{D}$ (i.e., a vertex with no edges directed into $v_{1}$ ). Let $\mathcal{D}^{-}$be the directed graph obtained by deleting $v_{1}$ from $\mathcal{D}$. Then

$$
\mathcal{P}\left(\mathcal{D}^{-}\right)=\lim _{x_{1} \rightarrow 1^{-}}\left(1-x_{1}\right) \mathcal{P}(\mathcal{D})
$$

Proof.

$$
\mathcal{P}(\mathcal{D})=\sum_{a_{1}=0}^{\infty} x_{1}^{a_{1}} \sum^{*} x_{2}^{a_{2}} \cdots x_{N}^{a_{N}}
$$

where "*" denotes that we are summing over all partitions associated with $\mathcal{D}^{-}$which have the added restriction that each $a_{i}$ associated with a vertex in $\mathcal{D}$ dominated by $a_{1}$ is, in fact, $\leq a_{1}$. Hence by Abel's Lemma [1, p. 190, Th. $14-7]$

$$
\lim _{x_{1} \rightarrow 1^{-}}\left(1-x_{1}\right) \mathcal{P}(\mathcal{D})=\lim _{a_{1} \rightarrow \infty} \sum^{*} x_{2}^{a_{2}} \cdots x_{N}^{a_{N}}=\mathcal{P}\left(\mathcal{D}^{-}\right),
$$

because any partition associated to $\mathcal{D}^{-}$will be counted once $a_{1}$ is large enough. 
4. $k$-diamonds with deleted source. Having established Theorem 7, the case of $k$-diamonds with deleted source is immediate from Theorem 6 .

Theorem 8. Now $X_{1}:=1$ and $X_{n}:=x_{2} \ldots x_{n}$. For $n \geq 2$ and $k \geq 1$,

$$
h_{n, k}^{*}=\prod_{j=2}^{(2 k+1) n+1} \frac{1}{1-X_{j}} \prod_{i=0}^{n-1} \prod_{l=1}^{k} \frac{1-X_{(2 k+1) i+(2 l-1)} X_{(2 k+1) i+(2 l+1)}}{1-X_{(2 k+1) i+(2 l+1)} / x_{(2 k+1) i+2 l}} .
$$

Proof. By Theorem 7,

$$
h_{n, k}^{*}=\lim _{x_{1} \rightarrow 1^{-}}\left(1-x_{1}\right) h_{n, k},
$$

and the desired result follows immediately once we observe that the denominator factor $1-X_{1}$ in $h_{n, k}$ is canceled by $1-x_{1}$.

Proof of Theorem 2. Theorem 2 is now an immediate consequence of Theorem 8 because in the above each $X_{j} \rightarrow q^{j-1}$.

5. Broken $k$-diamonds. As we noted in the introduction, the first line of Theorem 3 follows immediately by multiplying together the generating functions in Theorems 1 and 2 and letting $n \rightarrow \infty$. The exact formulations of the infinite products follow by algebraic simplification.

Proof of Theorem 4. We begin with the classic theta series identity $[1$, p. 178, Ex. 1]

$$
\sum_{n=-\infty}^{\infty}(-1)^{n} q^{n^{2}}=\frac{(q ; q)_{\infty}}{(-q ; q)_{\infty}}
$$

where

$$
(A ; q)_{\infty}=\prod_{j=0}^{\infty}\left(1-A q^{j}\right)
$$

By Theorem 3,

$$
\begin{aligned}
\left(\sum_{n=0}^{\infty} \Delta_{k}(n) q^{n}\right)\left(\sum_{n=-\infty}^{\infty}(-1)^{n} q^{n^{2}}\right) \\
\quad=\frac{(-q ; q)_{\infty}}{(q ; q)_{\infty}^{2}\left(-q^{2 k+1} ; q^{2 k+1}\right)_{\infty}} \cdot \frac{(q ; q)_{\infty}}{(-q ; q)_{\infty}}=\frac{1}{(q)_{\infty}\left(-q^{2 k+1} ; q^{2 k+1}\right)_{\infty}} \\
\left.=\frac{\left(q^{2 k+1} ; q^{4 k+2}\right)_{\infty}}{(q)_{\infty}} \quad \text { by }[1, \text { pp. } 164-165]\right) \\
=\prod_{\substack{n=1 \\
n \neq 2 k+1(\bmod 4 k+2)}}^{\infty} \frac{1}{1-q^{n}} .
\end{aligned}
$$


The first entry in the above sequence of equations clearly has

$$
\Delta_{k}(n)+2 \sum_{j=1}^{\infty} \Delta_{k}\left(n-j^{2}\right)(-1)^{j}
$$

as the coefficient of $q^{n}$, and the final entry is clearly the generating function for ordinary partitions in which no part is congruent to $2 k+1$ modulo $4 k+2$. Coefficient comparison concludes the proof of Theorem 4 .

Proof of Theorem 5.

$$
\begin{aligned}
\sum_{n=0}^{\infty} \Delta_{1}(n) q^{n} & =\frac{(-q ; q)_{\infty}}{(q ; q)_{\infty}^{2}\left(-q^{3} ; q^{3}\right)_{\infty}}=\frac{\left(q^{2} ; q^{2}\right)_{\infty}}{(q ; q)_{\infty}^{3}\left(-q^{3} ; q^{3}\right)_{\infty}} \\
& \equiv \frac{\left(q^{2} ; q^{2}\right)_{\infty}}{\left(q^{3} ; q^{3}\right)_{\infty}\left(-q^{3} ; q^{3}\right)_{\infty}}(\bmod 3) \\
& =\frac{\left(q^{2} ; q^{2}\right)_{\infty}}{\left(q^{6} ; q^{6}\right)_{\infty}} .
\end{aligned}
$$

The latter expression is clearly an even function of $q$. This means that the coefficients of odd powers of $q$ are all zero. Hence for all $n \geq 0$,

$$
\Delta_{1}(2 n+1) \equiv 0(\bmod 3)
$$

6. Conclusion. The culmination of our study led to an infinite family of modular forms. These, in turn, led to interesting arithmetic theorems and conjectures for the related partition functions. As we said in the introduction, Conjectures 1-3 suggest a true wealth of arithmetic theorems concerned with $\Delta_{k}(n)$.

We conclude with a remark that connects to previous work. Namely, in [10] we considered plane partitions with diagonals, i.e., the generating function of partitions into parts $a_{i}$ where the $a_{i}$ satisfy the order relations depicted in Fig. 6. As stated in [10, Thm. 1] its rational function repre-
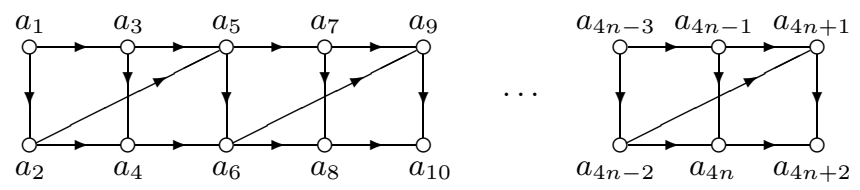

Fig. 6

sentation involves complicated irreducible numerator polynomials of total degree 2. We want to note that despite the nice structure of the rational function representation of $h_{n, k}^{*}$ in Theorem 4 above, the poset $H_{n, 2}^{*}$ can be viewed as a variation of the poset described by Figure 6 if drawn in an 
equivalent alternative to Figure 4 . For instance, for $n=3$ the poset $H_{3,2}^{*}$ can be depicted as in Figure 7.

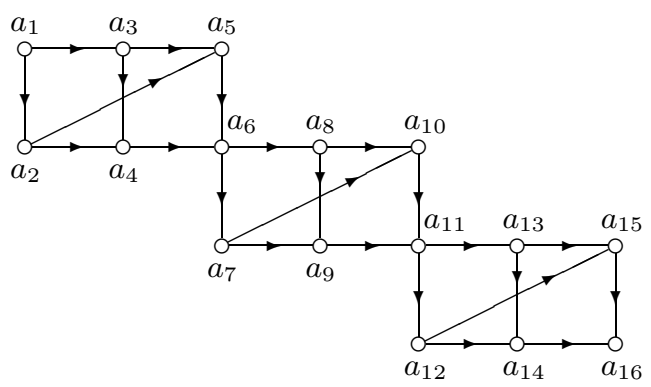

Fig. 7

Acknowledgements. We thank Ae Ja Yee and Song Heng Chan for their correction of Conjecture 3. In addition, we thank Manuel Kauers and Axel Riese for their help with the figures.

\section{References}

[1] G. E. Andrews, Number Theory, W. B. Saunders, Philadelphia, 1971. (Reprinted: Dover, New York, 1994.)

[2] —, MacMahon's partition analysis I: The lecture hall partition theorem, in: Mathematical Essays in Honor of Gian-Carlo Rota, Progr. Math. 161, Birkhäuser, Boston, 1998, 1-22.

[3] -, MacMahon's partition analysis II: Fundamental theorems, Ann. Comb. 4 (2000), $327-338$.

[4] G. E. Andrews and P. Paule, MacMahon's partition analysis IV: Hypergeometric multisums, Sém. Lothar. Combin. 42 (1999), B42i, 24 pp.

[5] G. E. Andrews, P. Paule, and A. Riese, MacMahon's partition analysis III: The Omega package, European J. Combin. 22 (2001), 887-904.

[6] - - - - MacMahon's partition analysis VI: A new reduction algorithm, Ann. Comb. 5 (2001), 251-270.

[7] -, - - - MacMahon's partition analysis VII: Constrained compositions, in: qSeries with Applications to Combinatorics, Number Theory, and Physics, B. C. Berndt and K. Ono (eds.), Contemp. Math. 291, Amer. Math. Soc., Providence, RI, 2001, 11-27.

[8] - - - - MacMahon's partition analysis VIII: Plane partition diamonds, Adv. in Appl. Math. 27 (2001), 231-242.

[9] - - - - MacMahon's partition analysis IX: $k$-gon partitions, Bull. Austral. Math. Soc. 64 (2001), 321-329.

[10] - - - - MacMahon's partition analysis $X$ : Plane partitions with diagonals, South East Asian J. Math. Math. Sci. 3 (2004), 3-14.

[11] G. E. Andrews, P. Paule, A. Riese, and V. Strehl, MacMahon's partition analysis $V$ : Bijections, recursions, and magic squares, in: Algebraic Combinatorics and Applications, A. Betten et al. (eds.), Springer, Berlin, 2001, 1-39. 
[12] S. Corteel and C. D. Savage, Plane partition diamonds and generalizations, Integers 3 (2003), A9, 8 pp.

[13] P. A. MacMahon, Combinatory Analysis, 2 vols., Cambridge Univ. Press, Cambridge, 1915-1916. (Reprinted: Chelsea, New York, 1960.)

Department of Mathematics

The Pennsylvania State University

University Park, PA 16802, U.S.A.

E-mail: andrews@math.psu.edu
Research Institute for Symbolic Computation Johannes Kepler University Linz 4040 Linz, Austria

E-mail: Peter.Paule@risc.uni-linz.ac.at

Received on 18.7.2006

and in revised form on 27.9.2006 